\title{
Budd-Chiari Syndrome: Two Cases with Different Courses
}

\author{
Shinjiro Inomata ${ }^{a}$ Yasuaki Takeyama ${ }^{a}$ Takashi Tanaka \\ Shu-ichi Ueda ${ }^{a}$ Daisuke Morihara ${ }^{a}$ b Shinya Nishizawa ${ }^{a, b}$ \\ Teruo Matsumoto ${ }^{a}$ Akira Anan $^{a, b}$ Hirokatsu Nishimura ${ }^{a}$ \\ Makoto Irie $^{a}$ Kaoru Iwata ${ }^{a}$ Satoshi Shakado a, b \\ Tetsuro Sohda ${ }^{a}$ Hideyuki Higashiharac \\ Masatoshi Okazakic Shotaro Sakisaka ${ }^{a}$ \\ aDepartment of Gastroenterology and Medicine and ${ }^{b}$ Division of Advanced \\ Clinical Research for Viral Hepatitis and Liver Cancer, Fukuoka University Faculty \\ of Medicine, and 'Department of Radiology, Fukuoka University School of \\ Medicine, Fukuoka, Japan
}

\section{Key Words}

Budd-Chiari syndrome - Antiphospholipid syndrome · Percutaneous transluminal angioplasty - Restenosis - Membranous obstruction of the inferior vena cava . Hepatocellular carcinoma

\begin{abstract}
We report two cases of Budd-Chiari syndrome. Case 1: A 57-year-old man presented with leg edema and esophageal varices. Cavography showed obstruction of the inferior vena cava with antiphospholipid syndrome. Further, the patient showed positive serology for hepatitis $C$ virus and consumed large quantities of alcohol. Percutaneous transluminal angioplasty was performed on this patient and anticoagulants administered; leg edema and esophageal varices were ameliorated although liver biopsy showed cirrhosis without evident congestion. More than 9 months since the diagnosis, restenosis of the inferior vena cava has not occurred. Case 2: A 73-year-old woman presented abdominal pain but no edema or varices. Cavography showed membranous obstruction of the inferior vena cava which required no therapy. Manifestation of portal hypertension was not present and liver function was maintained although liver biopsy showed obvious congestion. These cases showed untypical features against histopathology, and careful observation will be required for emergence of hepatocellular carcinoma.
\end{abstract}




\section{Introduction}

Budd-Chiari syndrome (BCS) is a rare disease characterized by the obstruction of hepatic venous outflow due to occlusion of the hepatic vein or the inferior vena cava (IVC). It leads progressively to sinusoidal congestion, ischemic injury to liver cells, portal hypertension and cirrhosis. The etiology of BCS is very diverse; $75 \%$ of cases are caused by both hereditary and acquired hypercoagulable states, and $25 \%$ of cases are idiopathic [1]. In Asian countries, most BCS cases are believed to be caused by membranous obstruction of the IVC (MOVC), while in Western countries, hepatic vein stenosis caused by coagulation disorder or neoplasm is a more common cause of the disease [2]. We report two patients with BCS due to IVC obstruction who presented with different etiologies, symptoms and untypical histopathologies and clinical courses (table 1).

\section{Case Reports}

\section{Case 1}

A 57-year-old man was admitted to our hospital because of distended abdomen, fatigue, and leg edema. He had a history of acute hepatitis at 18 years of age and bilateral leg thrombophlebitis at 27 years of age. He denied blood transfusion and a family history of liver disease, but he consumed large quantities of alcohol (145 g ethanol/day) and was a heavy smoker (40 cigarettes/day). Physical examination revealed a distended abdomen and leg edema with skin eruptions. Laboratory findings were as follows: platelet count $21.3 \times 10^{9} / \mathrm{l}$; albumin $17 \mathrm{~g} / \mathrm{l}$; aspartate aminotransferase $35 \mathrm{U} / \mathrm{l}$; alanine aminotransferase $12 \mathrm{U} / \mathrm{l}$; alkaline phosphatase $340 \mathrm{U} /$; gamma-glutamyltransferase $75 \mathrm{U} / \mathrm{l}$; total bilirubin $3.2 \mathrm{mg} / \mathrm{dl}$; prothrombin time $15.7 \mathrm{~s}$ (normal 11-14 s); activated thromboplastin time $36.2 \mathrm{~s}$ (normal 24-38 s); fibrinogen degradation products $28 \mu \mathrm{g} / \mathrm{ml}$ (normal $<5 \mu \mathrm{g} / \mathrm{ml}$ ); anticardiolipin beta 2glycoprotein I (anti-CL/beta 2-GPI) antibody $12.0 \mathrm{U} / \mathrm{ml}$ (normal $<3.5 \mathrm{U} / \mathrm{ml}$ ); lupus anticoagulant 1.04 (normal <1.30); positive hepatitis C virus (HCV) serology (HCV-RNA 1,810 kIU/l; serogroup 2) but not hepatitis B virus (HBV). Further, the patient was negative for markers of autoimmune hepatitis, primary biliary cirrhosis, Wilson's disease, and hemochromatosis.

Esophagogastric duodenoscopy (EGD) showed grade II esophageal varices, while ultrasonic cardiogram showed no abnormal findings. Abdominal computed tomography (CT) documented obstruction of the IVC, calcification above the obstructive part, extensive subcutaneous collateral veins, and dilated azygos and hemiazygos veins. Mild ascites and hepatosplenomegaly were also present. Cavography via the femoral vein revealed complete IVC obstruction above the orifice of the middle hepatic vein (MHV), and occlusion was not observed in the periphery of the hepatic vein. Further, it was observed that the dilated azygous venous system drained into the right atrium (fig. 1a). Since there was evident manifestation of portal hypertension and congestion, percutaneous transluminal angioplasty (PTA) was successfully performed with complete recanalization of the obstructed IVC (fig. 1b). Liver needle biopsy showed pseudolobules surrounded by thick fibrous connective tissue with moderate inflammatory infiltrates, indicating liver cirrhosis (fig. 2a). Then administration of heparin followed by warfarin was initiated. Six weeks after PTA, the leg edema improved and EGD showed that the esophageal varices had reduced to grade I. Restenosis of the IVC has not occurred more than 9 months since the treatment.

\section{Case 2}

A 73-year-old woman was admitted to our hospital for further investigation of abdominal pain and mild thrombocytopenia. She denied alcohol consumption, blood transfusion, and family history of liver disease. Physical examination revealed very mild tenderness at the right hypochondrium and mild hepatosplenomegaly but no leg edema. Laboratory studies demonstrated a platelet count of $10.1 \times 10^{9} / 1$, despite normal liver and coagulation function tests. Serology for hepatitis viruses and anticardiolipin antibody were all negative.

EGD and ultrasonic cardiogram showed no abnormal findings. Abdominal CT documented obstruction of the IVC, dilated azygos and hemiazygos veins, and extensive subcapsular collateral veins. 
Mild hepatosplenomegaly, ascites and an enlarged caudate lobe were also observed. Cavography via the femoral vein revealed complete obstruction of the IVC immediately under the diaphragm; the hepatic vein was not clearly visible although CT delineated the right hepatic vein (RHV) and MHV.

Additionally, cavography revealed dilated azygos venous system toward the right atrium (fig. 1c). Liver needle biopsy showed sinusoidal dilatation with congestion and irregular fibrosis (fig. 2 b). Percutaneous portal vein (umbilical portion) and MHV pressure measurements were simultaneously performed and were 19 and $15 \mathrm{~mm} \mathrm{Hg}$, respectively. These findings were consistent with BCS. Since laboratory studies presented normal liver and coagulation function tests and manifestation of portal hypertension was not evident, PTA was not performed and anticoagulants were not administered.

\section{Discussion}

BCS is a rare, heterogeneous, and potentially lethal condition related to the obstruction of the hepatic venous outflow tract [3]. Various etiologies of BCS are observed. The hypercoagulable state (antithrombin III deficiency, protein C/S deficiency, factor V Leiden mutation, prothrombin mutation, myeloproliferative disorders, paroxysmal nocturnal hemoglobinuria, antiphospholipid syndrome, pregnancy, and contraceptive use), tumor invasion, Behçet's disease, trauma, inflammatory bowel disease, and membranous obstruction comprise $75 \%$ of cases, and the remaining $25 \%$ of cases are idiopathic [1]. In Japan, it is generally believed that MOVC in BCS is caused by congenital malformation, although Kage et al. clarified that autopsy findings indicate that most cases of MOVC were caused by thrombosis [4].

In case 1, we diagnosed BCS due to a coagulation disorder since the patient had a history of thrombophlebitis 30 years before and exhibited positive serology for the anti-CL/beta 2-GPI antibody, indicating the presence of the antiphospholipid syndrome which is comparatively uncommon in Asian populations. Cavography showed IVC obstruction with a dilated azygos venous system also observed in case 2. However, in contrast to the case 2, case 1 had esophageal varices and leg edema. These findings were probably caused by HCV infection and consumption of large quantities of alcohol in addition to the IVC obstruction. Indeed, liver biopsy showed cirrhosis with moderate inflammation in the portal area, indicating viral hepatitis. Mallory body or inflammation in the central vein indicating alcoholic liver disease was not observed. Additionally, sinusoidal congestion was not evident.

After successful PTA and anticoagulant administration, the esophageal varices and leg edema gradually ameliorated, although liver biopsy suggested cirrhosis. However, PTA contains the risk of restenosis that possibly occurs in $17 \%$ of cases [5]. Qiao et al. reported a long-term effect of stent placement preventing restenosis; they found that restenosis after PTA and stent placement occurred in only 9.4\% cases [6]. Although the blood flow of the IVC has been maintained up to the present time in case 1, second PTA or stent placement will be possibly required hereafter. Additionally, long-term administration of an anticoagulant will be necessary to prevent restenosis $[7,8]$.

In case 2, a hypercoagulable state and other causes of BCS were not recognized; therefore, this case was diagnosed as MOVC. Although the patient presented with the classical triad of hepatomegaly, ascites, and abdominal pain, liver dysfunction and esophageal varices were not observed. Kamba et al. reported asymptomatic MOVC [9], showing complete IVC obstruction and collateral circulation from the RHV to the MHV; blood flow from the IVC drained into the right atrium via intrahepatic collaterals between the RHV and the MHV. There were no other collateral circulations such as the intercostal and the azygos venous systems, and the authors assessed that the intrahepatic shunt prevented the development of other collaterals and the emergence of various symptoms. 
The patient showed MOVC with extended subcapsular veins and a markedly dilated azygos venous system toward the right atrium but no intrahepatic shunt. Further, liver biopsy showed sinusoidal dilatation with congestion and irregular fibrosis indicating typical BCS. Therefore, various manifestations such as leg edema, esophageal varices, and liver dysfunction will be evident in the future. We speculate that low platelet count was due to hypersplenism, although maintained liver function was uncommon. Murad et al. reported that encephalopathy, ascites, prothrombin time, and bilirubin were independent determinants of survival rates, and overall survival rates at 1, 5 and 10 years were 82, 69 and $62 \%$, respectively [10]. The patient had ascites, so careful follow-up will be required.

It is important to note that the incidence of hepatocellular carcinoma in BCS patients is definitely high. Matsui et al. reported that the incidence was $25 \%$ and chronic liver congestion may contribute to hepatocarcinogenesis in MOVC [11]. Moreover, Okuda et al. emphasized the importance of the difference between primary hepatic vein thrombosis and obliterative disease predominantly affecting the hepatic portion of the IVC; the latter is frequently complicated by hepatocellular carcinoma, the former is not [12]. Therefore, the IVC obstruction could be a major predictor for hepatocellular carcinoma development. Our two cases showed obstruction of the IVC, and case 1 was serologically positive for HCV. Likewise, careful observation will be required.

In conclusion, we have described two cases of BCS. In one case obstruction of the IVC caused by a coagulation disorder was observed along with various manifestations that were ameliorated by PTA and anticoagulant administration although liver biopsy indicated cirrhosis; in the other case, MOVC with maintained liver function was observed and therapy was not required although liver biopsy indicated typical BCS.

Table 1. Comparison of the two cases

\begin{tabular}{lll}
\hline & Case 1 & Case 2 \\
\hline Sex & male & female \\
Age & 57 years & 73 years \\
Varices, leg edema & $(+)$ & $(-)$ \\
Alcohol consumption & $(+)$ & $(-)$ \\
Hepatitis virus & HCV $(+)$ & $(-)$ \\
Liver dysfunction & $(+)$ & $(-)$ \\
Etiology & antiphospholipid syndrome & MOVC \\
Obstructive part & IVC & IVC \\
Histopathology & pseudolobule with & sinusoidal dilatation with \\
& inflammation & congestion \\
Therapy & PTA, anticoagulants & $(-)$ \\
\hline
\end{tabular}

$\mathrm{HCV}=$ Hepatitis $\mathrm{C}$ virus; $\mathrm{MOVC}=$ membranous obstruction of the inferior vena cava; IVC = inferior vena cava; PTA = percutaneous transluminal angioplasty. 


\begin{tabular}{r|l|l|l}
$\begin{array}{r}\text { Case Reports in } \\
\text { Gastroenterology }\end{array}$ & $\begin{array}{l}\text { Case Rep Gastroenterol 2008;2:256-261 } \\
\text { D0I: 10.1159/000146063 }\end{array}$ & Published online: August 15, 2008 & $\begin{array}{l}\text { @ 2008 S. Karger AG, Basel } \\
\text { ISSN 1662-0631 } \\
\text { www.karger.com/crg }\end{array}$ \\
\hline
\end{tabular}

Fig. 1. a Cavography via the femoral vein. Complete obstruction of the IVC with the dilated azygos venous system drained into the right atrium. b Cavography via the femoral vein and internal jugular vein. PTA via the internal jugular vein and femoral vein recanalized the IVC. c Cavography via the femoral vein. The markedly dilated azygos venous system drained into the right atrium.
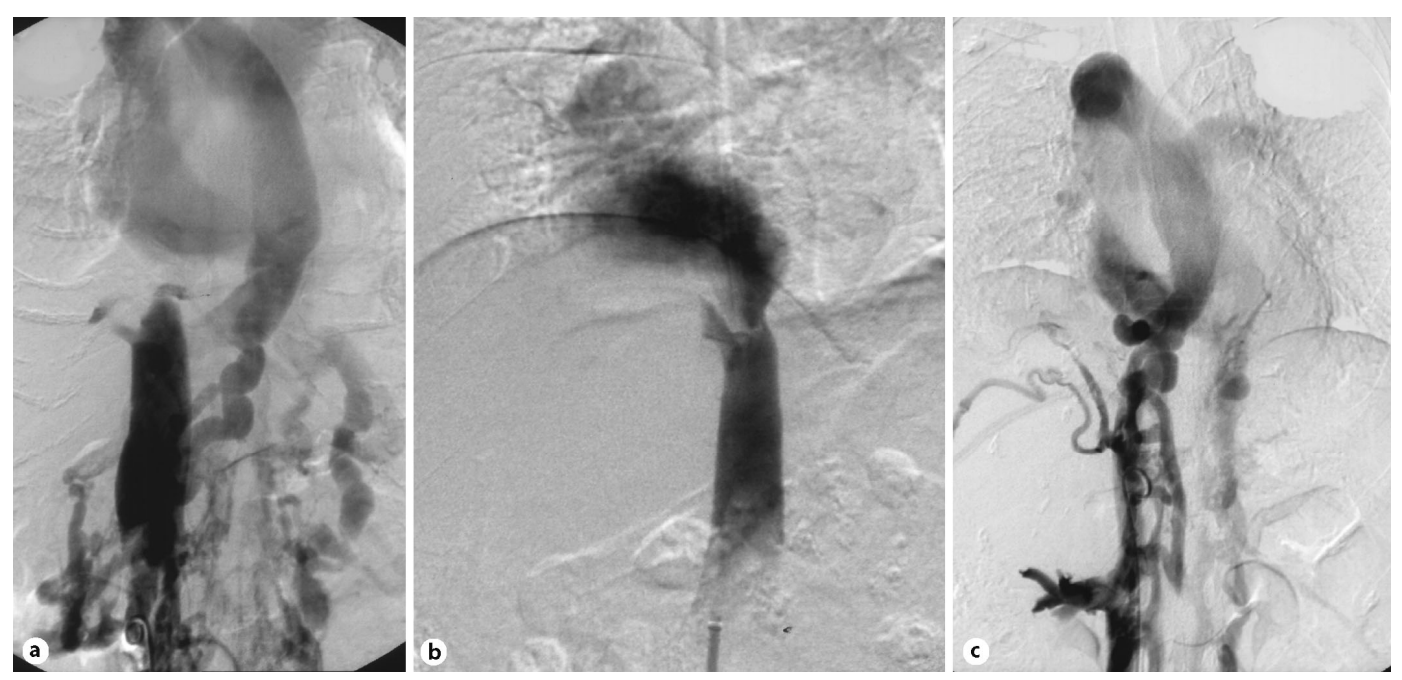

Fig. 2. a Liver biopsy. The section showed pseudolobules surrounded by thick fibrous connective tissue with moderate inflammatory cell infiltration. b Liver biopsy. The section showed sinusoidal dilatation with congestion and irregular fibrosis.
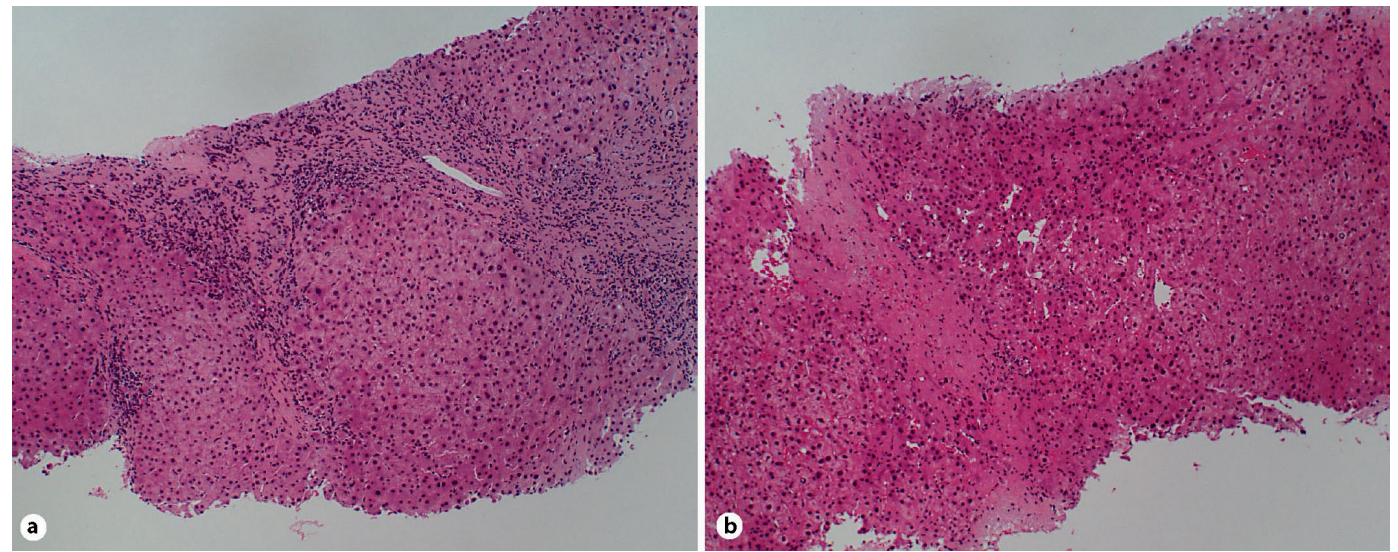


\section{References}

1 Menon KV, Shah V, Kamath PS: The Budd-Chiari syndrome. N Engl J Med 2004;350:578-585.

2 Faust TW, Sorrel MF: Budd-Chiari syndrome; in Schiff ER, Sorrell MF, Maddrey WC (eds): Schiffs Disease of the Liver, ed 8. Philadelphia, Lippincott-Raven, 1999, pp 1207-1213.

3 Valla DC: The diagnosis and management of the Budd-Chiari syndrome: consensus and controversies. Hepatology 2003;38:793-803.

-4 Kage M, Arakawa M, Kojiro M, Okuda K: Histopathology of membranous obstruction of the inferior vena cava in the Budd-Chiari syndrome. Gastroenterology 1992;102:2081-2090.

5 De BK, Biswas PK, Sen S, Das D, De KK, Das U, Mandal SK, Majumdar D: Management of the Budd-Chiari syndrome by balloon cavoplasty. Indian J Gastroenterol 2001;20:151-154.

-6 Qiao T, Liu CJ, Liu C, Chen K, Zhang XB, Zu MH: Interventional endovascular treatment of Budd-Chiari syndrome with long-term follow-up. Swiss Med Wkly 2005;135:318-326.

7 Levine JS, Branch DW, Rauch J: The antiphospholipid syndrome. N Engl J Med 2002;346:752-763.

-8 Espinosa G, Font J, Garcia-Pagan JC, Tassies D, Reverter JC, Gaig C, Cervantes F, Cervera R, Bosch J, Ingelmo M: Budd-Chiari syndrome secondary to antiphospholipid syndrome: clinical and immunologic characteristics of 43 patients. Medicine (Baltimore) 2001;80:345-354.

\9 Kamba M, Ochi S, Ochi H, Maruyama S, Sato H, Suto Y: Asymptomatic membranous obstruction of the inferior vena cava forming intrahepatic collateral pathways. J Gastroenterol 1995;30:783-785.

10 Murad SD, Valla DC, De Groen PC, Zaitoun G, Hopmans JA, Haagsma EB, Hoek B, Hansen BE, Rosendaal FR, Janssen H: Determinants of survival and the effect of portosystemic shunting in patients with Budd-Chiari syndrome. Hepatology 2004;39:500-508.

11 Matsui S, Ichida T, Watanabe M, Sugitani S, Suda T, Takahashi T, Asakura H: Clinical features and hepatocellular carcinoma arising in patients with membranous obstruction of the inferior vena cava: in reference to hepatitis viral infection. J Gastroenterol Hepatol 2000;15:1205-1211.

12 Okuda K, Kage M, Shrestha S: Proposal of a new nomenclature for Budd-Chiari syndrome: Hepatic vein thrombosis versus thrombosis of the inferior vena cava at its hepatic portion. Hepatology 1998;28:1191-1198. 